\title{
Collisional Relaxation of Fast Electrons Energy in Gas
}

\author{
Alexander Metel $^{1, *}$, Enver Mustafaev ${ }^{1}$, Yury Melnik ${ }^{1}$, and Khaled Hamdy ${ }^{2}$ \\ ${ }^{1}$ Moscow State Technological University "STANKIN", RU-127055, Moscow, Russia \\ ${ }^{2}$ Production Engineering and Mechanical Design Department, Faculty of Engineering, Minia University, EG-61519, Minia, Egypt
}

\begin{abstract}
We present results of theoretical and experimental study of collisional relaxation of fast electrons energy in gas. The dependence on the gas pressure $p$ and electron energy $\varepsilon$ of the mean pass $\Lambda$ of fast electrons injected into a gas being sufficient to spend on ionization all their initial energy $\varepsilon$ has been calculated. It was found that $\Lambda$ is directly proportional to $\varepsilon^{2}$ and inversely proportional to the gas pressure. To sustain glow discharge with electrostatic confinement of fast electrons, $\Lambda$ should be less than the mean way to the anode of emitted by the cathode electrons.
\end{abstract}

\section{Introduction}

To transport accelerated particles and atoms of deposited materials from the particles sources to the surface of the workpiece in the working vacuum chamber, the length of their free path must exceed the size of the workpiece $10 \mathrm{~cm}$, and therefore these particles are obtained using a high-frequency [1], vacuum arc [2] and magnetron [3] discharges at a gas pressure below $\sim 0.1 \mathrm{~Pa}$. To fill a large working volume with a homogeneous plasma, a discharge with thermionic cathodes and a peripheral magnetic field on the surface of the chamber, is also used [4]. In this case, a cylindrical hollow cathode with an inner diameter of several millimeters made of a refractory metal is often used as a thermionic cathode [5]. As for the glow discharge with a cold hollow cathode [6], the range of its applications for surface treatment was limited by the relatively high working gas pressure. There were no data in the literature on a cold cathode glow discharge at a gas pressure below $1 \mathrm{~Pa}$ [714]. There were no well-established ideas about the factors that determine the value of its lower working pressure [14-16] and the mechanism of the discharge. There was also no general opinion about the physical processes causing the effect of the hollow cathode, which reflects back electrons emitted from the plasma enclosed inside it. At the same time, the results of research and practical application of the discharge indicated that a glow discharge with a hollow cathode is capable of forming ion and electron beams [17-20], as well as a dense and homogeneous plasma throughout the volume of the cathode hollow.

Most researchers explained that the extinction of the hollow cathode glow discharge is caused by the loss of electrons emitted by the cathode with a decrease in the gas pressure. either as a result of their absorption by the cathode surface or their escape from the cathode [21-23]. In the second case, the lower pressure limit of the discharge should depend on the ratio between the average path length $L$ of electrons inside the cathode before escaping from the cathode and the energy relaxation length $\Lambda$ hereinafter referred to as the average path length of a fast electron, which it has to pass in order to spend all its energy on the gas ionization. When $\Lambda$ exceeds $L$, the electrons spend only part of the energy on the gas ionization inside the cathode. Therefore, with increasing $\Lambda$, the ionization intensity in the cathode decreases and the discharge extinguishes.

\section{Beams produced by a flat grid}

In discharges with electrostatic traps at low gas pressure, electrons emitted by the cathode pass through the cathode sheath without collisions and enter the plasma with an energy of $\varepsilon_{0}=e U$ from hundreds to thousands of $\mathrm{eV}$, corresponding to the cathode fall of potential $U$. Each of them travels a path exceeding the size of the discharge space by hundreds and thousands times and forms tens of new free electrons inside the trap. Here it is necessary to trace the entire path of a fast electron and determine how many new electrons and with what energy it forms both in equipotential plasma and in electric fields of space charges.

To determine $\Lambda$ we use a well-known experimental fact: the number $N$ of free electrons produced by a fast electron injected into a gas is directly proportional to its initial energy. The ionization cost $W$ of a new electron is constant for any initial energy of the fast electron and is equal to $46 \mathrm{eV}$ for helium, $37 \mathrm{eV}$ for neon, $26 \mathrm{eV}$ for argon, $24 \mathrm{eV}$ for krypton, $37 \mathrm{eV}$ for nitrogen, $36 \mathrm{eV}$ for hydrogen, and $33 \mathrm{eV}$ for oxygen [24]. This allows us to abstract from the details of real events occurring with the electron and restrict ourselves to formalism, which considers only its ionization collisions, in which on formation of a new electron is spent not the ionization energy $E_{\mathrm{i}}$, but the ionization cost $W$. To determine the electron mean free path between such collisions, we will

Corresponding author: a.metel@stankin.ru 
use the experimental values of the ionization cross section $\sigma$ given in the monograph by Massey and Barhop [25].

As the distance $d x$ travels, the number $N_{0}$ of electrons with the initial energy $\varepsilon$ decreases as a result of ionizing collisions:

$$
\mathrm{d} N_{0}=-N_{0} \mathrm{~d} x / \lambda_{0},
$$

where $\lambda_{0}=1 / n \sigma(\varepsilon), n$ is the density of gas molecules, and $\sigma(\varepsilon)$ is the dependence of the ionization cross section on the electron energy $\varepsilon$. After the formation of a new free electron, the initial energy $\varepsilon$ of a fast electron decreases on average by $W$. Electrons with energy $(\varepsilon-W)$ appear, and the number of these electrons $N_{1}$, as they travel the distance $\mathrm{d} x$, changes by

$$
\mathrm{dN}_{1}=-N_{1} \mathrm{~d} x / \lambda_{1}+\mathrm{N}_{0} \mathrm{~d} x / \lambda_{0}
$$

where $\lambda_{1}=1 / n \sigma(\varepsilon-W)$. After $\mathrm{j}$ collisions, the electron energy decreases to $\varepsilon-\mathrm{j} W$, and the number $N_{\mathrm{j}}$ of electrons with indicated energy changes with an increase by $\mathrm{d} x$ in the path by

$$
\mathrm{d} N_{\mathrm{j}}=-N_{\mathrm{j}} \mathrm{d} x / \lambda_{\mathrm{j}}+N_{\mathrm{j}-1} \mathrm{~d} x / \lambda_{\mathrm{j}-1},
$$

where $\lambda_{\mathrm{j}}=1 / n \sigma(\varepsilon-\mathrm{j} W)$ and $\mathrm{j}=1,2, \ldots,(N-1)$. After $N$ collisions, the electron energy becomes less than $W$, and it can no longer ionize the gas.

Let us consider ionization of argon by electrons with an energy of $4 \mathrm{keV}$. After the formation of the first 20 free electrons the energy of each electron decreases on average by $20 W \approx 520 \mathrm{eV}$, i.e., from 5 to $3.48 \mathrm{keV}$. The ionization cross section with the indicated decrease in energy varies within the range $\sigma=(3.5 \pm 0.2) \times 10^{-17} \mathrm{~cm}^{2}$. Therefore, on the path length $x$ corresponding to a given change in energy, the value $\lambda=1 / n \sigma$ can be assumed to be approximately constant. In this case, the solution of the equations (1) and (3) gives the following expressions for the dependence of the number $N_{\mathrm{j}}$ of electrons with an average energy $(\varepsilon-\mathrm{j} W)$ on the relative length $y=x / \lambda$ of the traversed path

$$
N_{\mathrm{j}}(y)=N_{0}\left(\mathrm{y}^{\mathrm{j}} / \mathrm{j} !\right) \exp (-y) .
$$

The total number of electrons with energy $\varepsilon \geq \varepsilon-\mathrm{kW}$ after their path $x=y \lambda$ is equal to $N_{0} M_{k}(y)$, where

$$
M_{\mathrm{k}}(y) \equiv \sum_{j=0}^{k} \frac{y^{j}}{j !} \exp (-y)
$$

Figure 1 shows the dependences of the relative number $N_{\mathrm{j}}(\mathrm{y}) / N_{0}$ of electrons with different energies from $\varepsilon-20 W$ to $\varepsilon$ on the length of the traversed path $y$, and Figure 2 - fractions $M_{\mathrm{k}}(\mathrm{y})$ of electrons with energy $\varepsilon$ $\geq \varepsilon-\mathrm{k} W$.

Figure 3 shows the energy distributions of electrons after they have traveled a path of $5 \lambda, 10 \lambda$ and $20 \lambda$.

The envelopes of discrete spectra in Fig. 3 present the energy distribution of electrons, which traversed a path of the same length. After passing the distance $\mathrm{k} \lambda$, the electron energy can have different values in the range from $\varepsilon-(3 \mathrm{k} / 2) W$ to $\varepsilon-(\mathrm{k} / 2) W$, but the energy $\varepsilon-\mathrm{k} W$ is most likely. This means that passing $\mathrm{k} \lambda$, it produces $\mathrm{k}$ new free electrons.

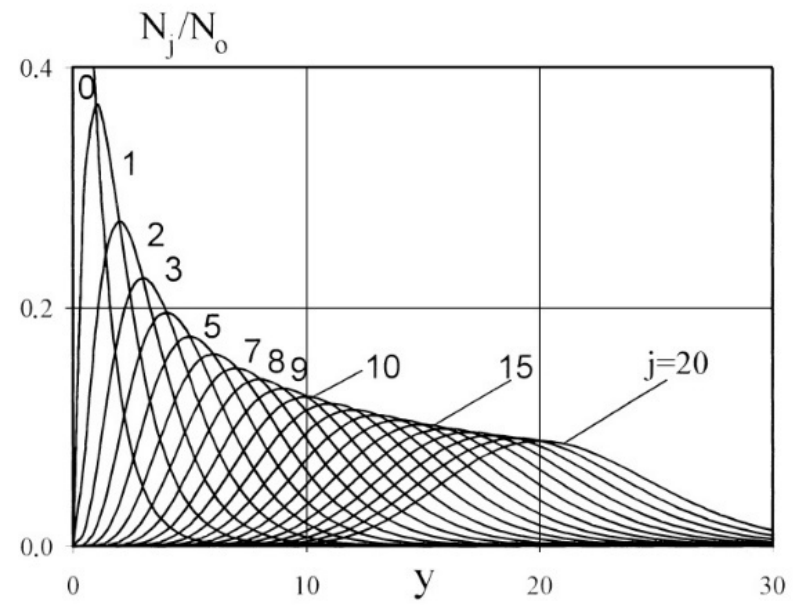

Fig. 1. Relative numbers $N_{\mathrm{j}}(\mathrm{y}) / N_{0}$ of electrons versus the length of the traversed path $y$.

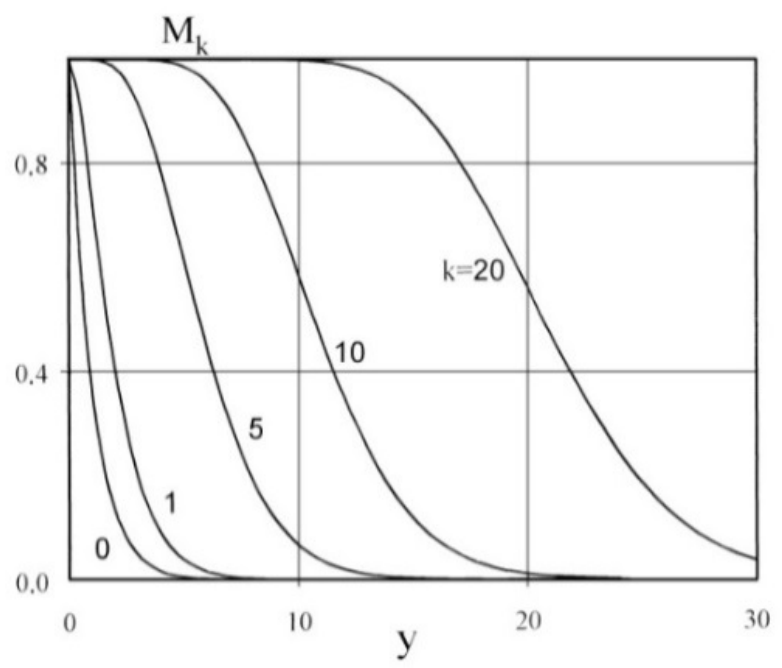

Fig. 2. Fractions $M_{\mathrm{k}}$ (y) of electrons with energy $\varepsilon \geq \varepsilon-\mathrm{kW}$.

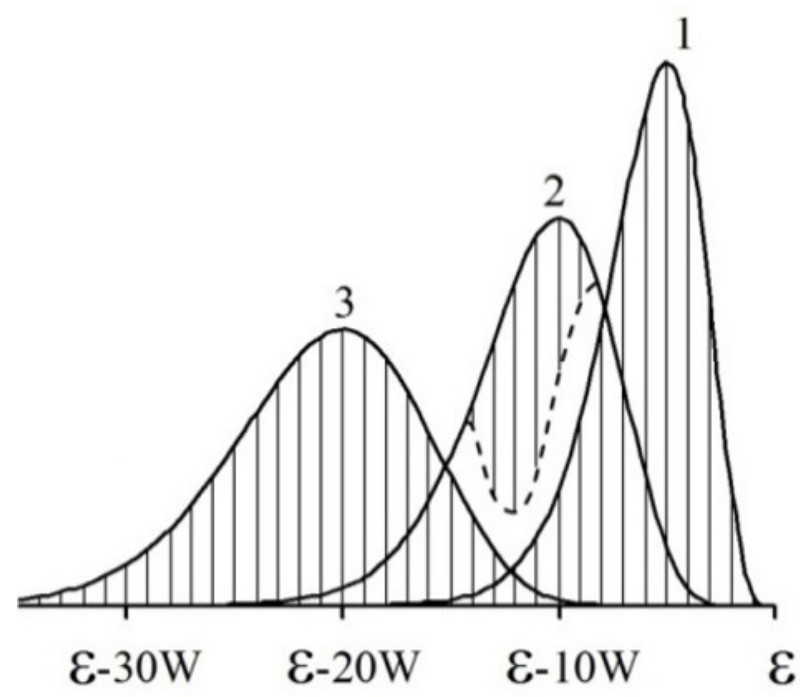

Fig. 3. Energy distributions of electrons traveled a path of $5 \lambda$

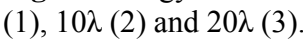


This conclusion is valid only under the condition that the electron energy does not go beyond the range in which the approximation of $\lambda$ constancy is permissible.

After passing the distance $\mathrm{k} \lambda / 2$, the energy of all electrons does not really go out of the range from $\varepsilon-\mathrm{k} W$ to $\varepsilon$ (Fig.2), however, after the path $\mathrm{k} \lambda$ the number of such electrons diminishes by about two times. Therefore, for $\mathrm{k}=20$ we can quite reasonably represent only the initial section of the path with a length of $10 \lambda$ as a sequence of 10 segments of the path, each with a length of $\lambda=1 / n_{0} \sigma$ and suppose that at the end of each segment a new free electron appears and the energy of the fast electron decreases by $W$.

To reduce the error with a further increase in the length of its path, we must abandon the constancy of $\lambda$ and assume that the first electron appears at the end of a segment with the length of $\lambda_{0}=1 / n_{0} \sigma(\varepsilon)$, the second electron appears at the end of a segment with the length of $\lambda_{1}=1 / n_{0} \sigma(\varepsilon-\mathrm{W})$, at which the energy of the fast electron is equal to $(\varepsilon-W)$, the third electron appears at the end of a segment with the length of $\lambda_{2}=1 / n_{0} \sigma(\varepsilon-$ $2 \mathrm{~W}$ ), at which the energy of the fast electron is equal to $(\varepsilon-2 W)$, at which the fast electron energy is equal to $(\varepsilon-$ $2 \mathrm{~W}$ ), etc. As a result, the electron passes a sequence of $N$ segments and at the end of each of them forms a new free electron and loses energy of $W$. In total, the electron forms $N=\varepsilon / W$ of new free electrons and passes the path

$$
\Lambda(\mathrm{p}, \varepsilon)=N \lambda_{\mathrm{N}}(\mathrm{p}, \varepsilon)
$$

where $\lambda_{N}(p, \varepsilon)$ is the path $\lambda_{\mathrm{j}}$ between two successive ionizing collisions, averaged over the entire range of the electron energy from $\varepsilon$ to $W$

$$
\begin{aligned}
& \lambda_{\mathrm{N}}(\mathrm{p}, \varepsilon)= \\
= & \frac{1}{n_{\mathrm{o}}(p)(\varepsilon-W)} \int_{W}^{\varepsilon} \frac{\mathrm{d} \varepsilon}{\sigma(\varepsilon)} \frac{1}{n_{\mathrm{o}}(p)(N-1)} \int_{1}^{N} \frac{\mathrm{d} z}{\sigma\left(W_{z}\right)}
\end{aligned}
$$

The dashed lines in Figure 4 show the dependence of the argon ionization cross-section on the electron energy [25], the dashed-dotted lines show the mean free path $\lambda_{0}$ of an electron with energy $\varepsilon$ between ionizing collisions, and the solid lines shows the dependence on the initial energy $\varepsilon=W N$ of the electron path between ionizing collisions $\lambda_{N}$ calculated by formula (7) at room temperature $293 \mathrm{~K}$ and argon pressure $1.0 \mathrm{~Pa}\left(n_{0}=\right.$ $\left.2.5 \times 10^{14} \mathrm{~cm}^{-3}\right)$.

At the electron energy $\varepsilon$ ranging from 40 to $400 \mathrm{eV}$ the average electron path between ionizing collisions $\lambda_{N}$ can be supposed independent of $\varepsilon$ and the energy relaxation length $\Lambda(\mathrm{p}, \varepsilon)=(\varepsilon / W) \lambda_{N}(p, \varepsilon)$ is directly proportional to the energy $\varepsilon$.

At the electron energy $\varepsilon$ exceeding $1 \mathrm{keV}$ the average electron path between ionizing collisions $\lambda_{N}$ can be supposed proportional to $\varepsilon$ and the energy relaxation length $\Lambda(\mathrm{p}, \varepsilon)=(\varepsilon / W) \lambda_{\mathrm{N}}(\mathrm{p}, \varepsilon)$ is proportional to $\varepsilon^{2}$.
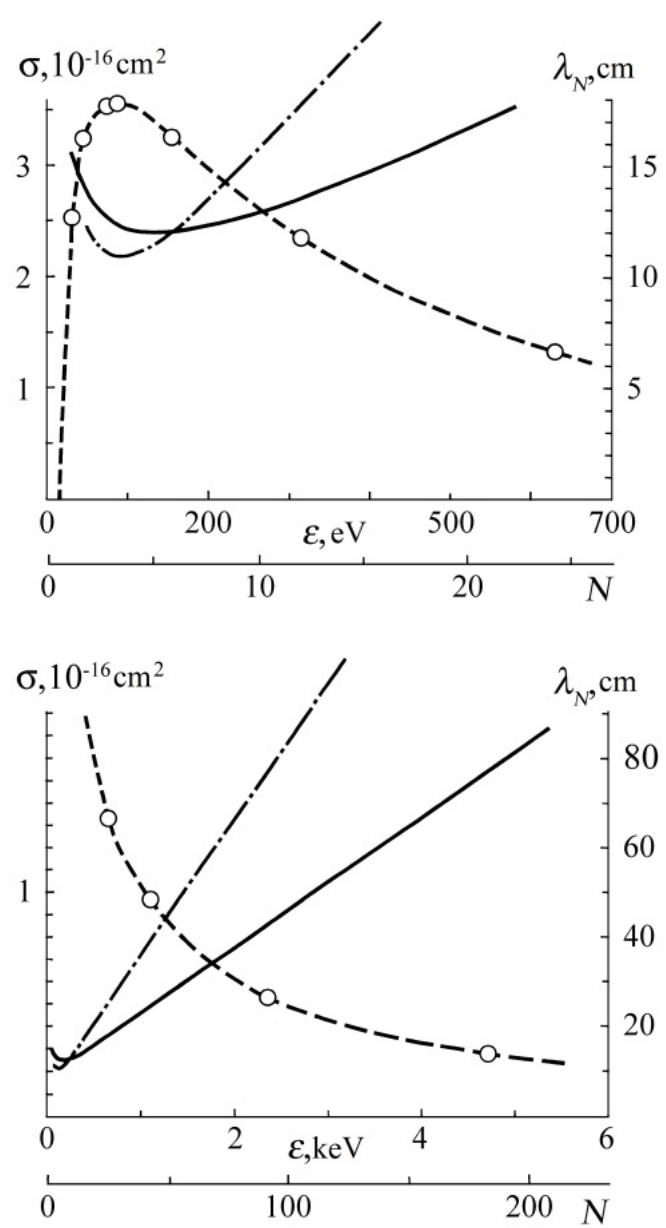

Fig. 4. Dependence on the electron energy $\varepsilon$ of the argon ionization cross-section (dashed lines), the mean free path $\lambda_{0}$ of an electron with energy $\varepsilon$ between ionizing collisions (dasheddotted lines) and the average electron path between ionizing collisions $\lambda_{\mathrm{N}}$ at room temperature $293 \mathrm{~K}$ and argon pressure 1.0 $\mathrm{Pa}$ (solid lines).

\section{Electrostatic traps}

Figure 5 presents a schematic of the most wide-spread electrostatic traps based on hollow cathodes featuring trajectory in discharge plasma 1 of electron 2 emitted by the cathode 3 bombarded by ion 4 . This electron is hundreds times reflected in a space charge sheath 5 by the voltage of power supply 6 from all parts of the cathode surface enveloping the trap. For this reason distribution of the electron velocity in all the traps is quite isotropic. The distance traveled by electrons before escaping from the trap volume $V$ through aperture of losses $S_{\mathrm{o}}$ is equal to

$$
L=4 V / S_{0}
$$

and can by hundreds times exceed the trap width.

Numerous reflections of emitted by the cathode electrons in the cathode sheath ensure an adequate distance traveled by these electrons and, hence, sustaining of the discharge at low pressure. 


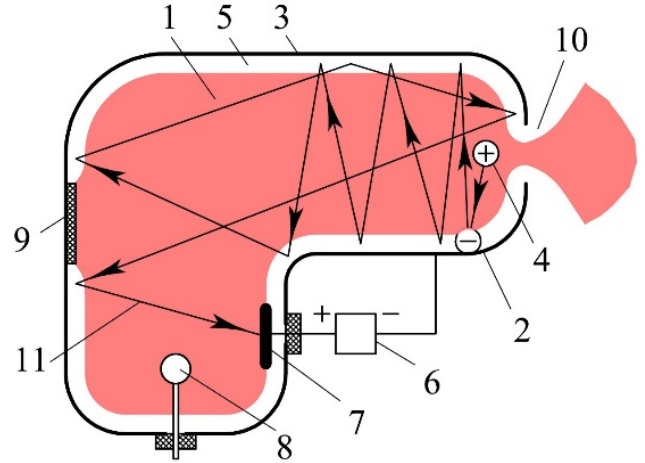

Fig. 5. Electrostatic trap shaped as a hollow cathode.

On their long way to the anode emitted by the cathode electrons visit all parts of the trap and for this reason they are distributed quite homogeneously. Probability of the gas ionization by those electrons and the plasma density are also quite uniform. The lower pressure limit of the discharge should depend on the ratio between the average path length $L$ of electrons inside the cathode before escaping from it and the energy relaxation length $\Lambda$. Hereinafter the latter is referred to as the average path length of a fast electron, during the passage of which its energy decreases to the ionization threshold $E_{\mathrm{i}}$. When the energy relaxation length $\Lambda$ exceeds $L$, fast electrons spend only part of their energy on ionizing the gas inside the cathode. To compensate the electron losses it is needed to produce fast electrons in the cathode sheath. At an unchanged discharge current for an increase in the number of additional fast electrons from the sheath, it is needed to increase its width. In accordance with the Child-Langmuir law the sheath width grows with the cathode fall of potential. This is the reason for the discharge voltage increase with the pressure decreasing. 6.

Another type of electrostatic trap is shown in Figure

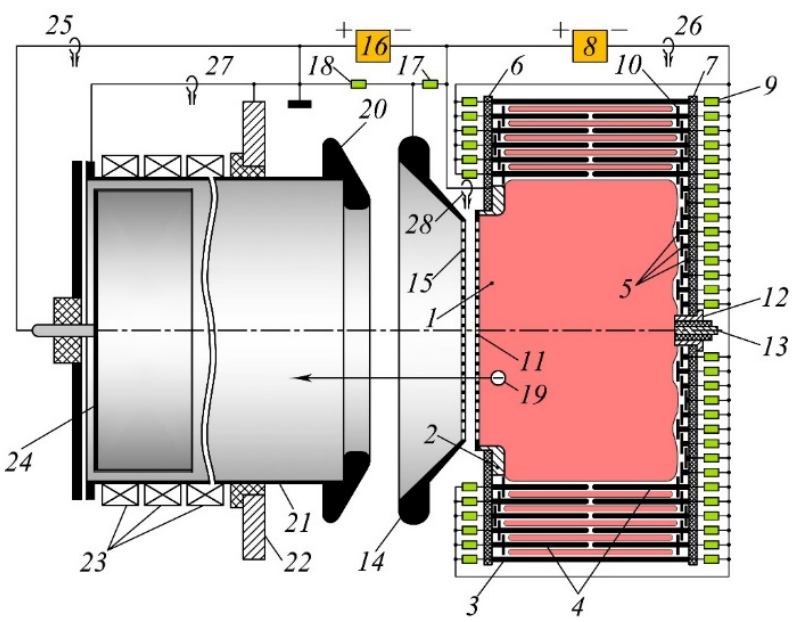

Fig. 6. Schematic of an electron gun with plasma emitter generated by the glow discharge with a multirod cathode.

The plasma emitter 1 of electrons is produced by the glow discharge between anode 2 and a multirod cold cathode. The cathode comprises an outer hexagonal envelope composed of 204 parallel 5-mm-diameter and 200-mm-long cathode rods 3, which are spaced by 1.5 $\mathrm{mm}$, and located inside the envelope hexagonal layers of 5-mm-diameter and 98-mm-long cathode rods 4 the distance between their axes amounting to $15 \mathrm{~mm}$, as well as 23-mm-diameter and 1-mm-thick cathode discs 5.

All the cathode rods and discs are fastened on two dielectric flanges 6 and 7, they are isolated from each other and connected to the negative pole of power supply 8 through resistors 9 with $430-\Omega$ resistance. To prevent from a contact of the dielectric flanges 6 and 7 with the plasma filling the gaps between rods 4 , each rod has a 23-mm-diameter and 1-mm-thick metal disk 10. Disks 10 and discs 5 are placed in three layers with a distance of $1 \mathrm{~mm}$ between them.

Eight hexagonal layers are formed with 1104 inner rods, and diameter of emissive grid 11 fastened on the anode 2 is equal to $110 \mathrm{~mm}$. A photograph of this anode and inner rods with discs mounted on dielectric flange 6 is presented in Figure 7. In the center of opposite flange 7 is positioned an igniting arrangement with coaxial electrodes 12 and 13. After between those electrodes a high-voltage pulse is applied, the central part of the trap, which is free of rods, is filled with pulsed arc plasma.

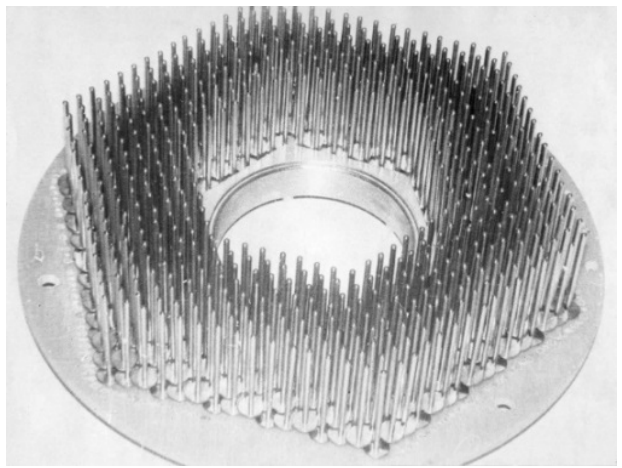

Fig. 7. Photograph of a multirod electrostatic trap.

Dielectric flanges are fixed to each other with three steel spacers, which are also used to fasten resistors 9 . The distance between emissive grid 11 and accelerating grid 15 , which is connected to focusing electrode 14 , amounts to $13 \mathrm{~mm}$. Both grids are 1-mm-thick and have 85 pairs of aligned 6-mm-diameter apertures, distance between aperture centers amounts to $10 \mathrm{~mm}$.

As an accelerating voltage power supply is used a high-voltage square pulse generator 16 based on pulse forming networks. A time delay between switching on the networks allows a decrease of the pulse top ripple down to $1 \%$. Generator 16 with $20-\Omega$ impedance induces 200- $\mu$ s-wide high-voltage pulses with amplitude up to $U$ $=500 \mathrm{kV}$ across an active load composed of connected in series resistors 17 with $2-\Omega$ resistance and 18 with 18 $\Omega$ resistance. Electrons 19 are accelerated in the gap between grids 11 and 15 by the voltage drop across resistor 17 up to energy of $0.1 \mathrm{eU}$ and then they are accelerated in the gap between focusing electrode 14 and grounded electrode 20 by the voltage drop across resistor 18 up to energy of $e U$. 
Electrode 20 is fastened on 0.8-m-long electron beam track 21, which is isolated from lateral flange 22 of the vacuum chamber. Displacement of track 21 in horizontal direction relative to flange 22 makes it possible to vary the distance between electrode 20 and grid 15 from 70 to $120 \mathrm{~mm}$. To move in vertical direction electrode 14 together with the whole emissive assembly, which is fastened to a segmented condenser-type high-voltage feedthrough, a special positioner is used, which is mounted inside the central segment of the feedthrough. The positioner also enables canting angle variation of the assembly axis and rotation of the assembly around the vertical axis of the vacuum chamber.

Outside the chamber on track 21 (Fig. 6) are mounted solenoids 23 producing magnetic field with induction up to $0.02 \mathrm{~T}$. At the track end is mounted collector 24 intended to measure using Rogowski coil 25 the current of electron beam, which passed along the track. Current pulses in the circuits of multirod cathode, track 21 and anode 2 are measured, respectively, with Rogowski coils 26, 27 and 28.

The plasma emitter of electrons produced using glow discharge with a multirod electrostatic trap made it possible to produce pulsed beams with homogeneous distribution of 700-A current of $300-\mathrm{keV}$ electrons over 170-mm-diameter cross-section of the beam at the pulse width of $\sim 200 \mu \mathrm{s}$. Connection of about one thousand cathode rods to the discharge power supply through individual resistors prevents the glow discharge with up to $1 \mathrm{kA}$ current from transition to arc and thus ensures homogeneity of the plasma emitter.

At one and the same discharge current $I$ in the cathode circuit the beam current $I_{b}$ in the circuit of collector is rising with increasing the accelerating voltage and stops rising at $I_{b}=I$, when all electrons produced in the discharge enter the accelerating gap. At the maximal beam current $700 \mathrm{~A}$ of $300-\mathrm{keV}$ electrons the energy capacity of the beam at the pulse width of 200 $\mu$ s amounts to $40 \mathrm{~kJ}$ and at the beam cross-section area of $0.025 \mathrm{~m}^{2}$ the surface density of energy absorbed by the collector bottom exceeds $10^{6} \mathrm{~J} / \mathrm{m}^{2}$. The results obtained overwhelm those previously presented anywhere.

The above broad pulsed beams based on collisional relaxation of fast electrons energy in the plasma emitters of beam and plasma sources were successfully applied for the surface modification of the machinery products. There are many other technological systems based on collisional relaxation of fast electrons energy, for instance [26-29].

\section{Acknowledgments}

This work was supported by the Russian Science Foundation under grant 20-19-00620. This work was carried out using equipment provided by the Center of Collective Use of MSUT "STANKIN".

\section{References}

1. K. Suzuki, S. Okudaira, N. Sakudo, Jap. J. Appl. Phys., 16, 1979 (1977)

2. Handbook of vacuum arc science and technology: fundamentals and applications, Eds by R. L. Boxman, Ph. J. Martin, D. Sanders (Park Ridge, New Jersey, U.S.A.: Noyes Publications, 1995)

3. A. Anders, Surf. Coat. Technol., 205, 51 (2011)

4. M. Benhassine, Revue Phys. Appl., 19, 545 (1984)

5. D.J. Willins, R.L.F. Boyd, J. Phys. D: Appl. Phys., 6, 1447 (1973)

6. B.I. Moskalev, Discharge with a hollow cathode. (Moscow: Energy, 1969) [in Russian]

7. A. Güntherschulze, Zeitschr. für techn. Phys., 11, 49 (1930).

8. T. Musha, J. Phys. Soc. Japan, 17, 1440 (1962)

9. C. Popovici, M. Somesan, Appl. Phys. Letters, 8, 103 (1966).

10. M. Yamashita, M. Kimura, Jap. J. Appl. Phys., 19, L449 (1980)

11. K. Danzmann, M. Kock, J. Phys. B: At. Mol. Phys., 14, 2989 (1981)

12. I. Iova, I. Chera, M. Ganciu-Petcu, Rev. Roum. Phys., 29, 519 (1984).

13. E.M. Veldhuizen F.J. Hoog, J. Phys. D: Appl. Phys., 17, 953 (1984)

14. W. Stephenson, Phil. Magazine., 2, 556 (1926)

15. H. Helm, Zeitschrift Naturforsch., 27a, 1812 (1972)

16. Yu.M. Kagan, J. Phys. D: Appl. Phys., 18, 1113 (1985)

17. H.L.L. Paassen, E.C. Muly, R.J. Allen, Proc. of the National Electronics Conference., 18, 590 (1962)

18. H.L.L. Paassen, E.C. Muly, R.J. Allen, Proc. of the National Electronics Conference., 18, 597 (1962)

19. D. Bloess, I. Kamber, H. Riege, Nucl. Instrum. and Meth., 205, 173 (1983)

20. J. Mizeraczyk, M. Neiger, Appl. Phys., B33, 17 (1984)

21. A. Metel, S. Grigoriev, Y. Melnik, M. Volosova, E. Mustafaev, Machines, 8, 42 (2020)

22. A.S. Metel, S.N. Grigoriev, Yu.A. Melnik, V.P. Bolbukov, Instrum. Exp. Tech., 55, 288 (2012)

23. A. Metel, V. Bolbukov, M. Volosova, S. Grigoriev, Yu. Melnik, Instrum. Exp. Tech., 57(3), 345 (2014)

24. A. Dalgarno, Atomic and Molecular Processes, ed. D.R. Bates (Academic Press, New York, 1962)

25. H.S.W. Massey, E.H.S. Burhop, Electronic and Ionic Impact Phenomena (Oxford: Clarendon press, 1952)

26. M. Volosova, S. Grigoriev, A. Metel, A. Shein, Coatings, 8, 287 (2018)

27. S.N. Grigoriev, Yu.A. Melnik, A.S. Metel, V.V. Panin, Instrum. Exp. Tech., 52, 602 (2009)

28. S. Grigoriev, A. Metel, NATO Science Series, Series II: Mathematics, Physics and Chemistry, 155, 147 (2004) 
29. A. Metel, S. Grigoriev, Yu. Melnik, V. Panin, V. Prudnikov, Jap. J. Appl. Phys., 50, 08JG04 (2011) 